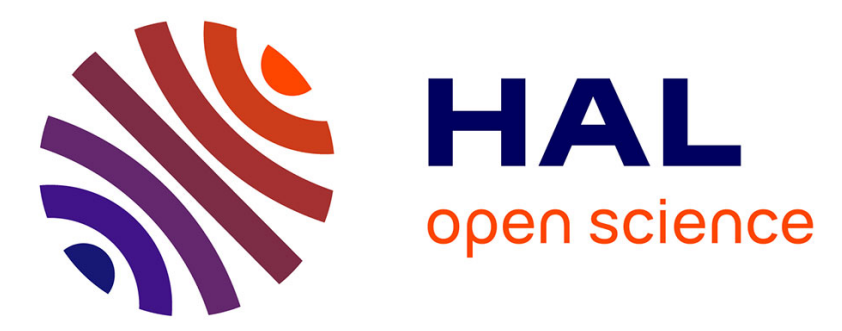

\title{
A data-driven temperature compensation approach for Structural Health Monitoring using Lamb waves
}

Claude Fendzi, Marc Rebillat, Nazih Mechbal, Mikhail Guskov, Gérard

Coffignal

\section{- To cite this version:}

Claude Fendzi, Marc Rebillat, Nazih Mechbal, Mikhail Guskov, Gérard Coffignal. A data-driven temperature compensation approach for Structural Health Monitoring using Lamb waves. Structural Health Monitoring, 2016, 15 (5), pp.525-540. 10.1177/1475921716650997 . hal-01376868

\section{HAL Id: hal-01376868 \\ https://hal.science/hal-01376868}

Submitted on 5 Oct 2016

HAL is a multi-disciplinary open access archive for the deposit and dissemination of scientific research documents, whether they are published or not. The documents may come from teaching and research institutions in France or abroad, or from public or private research centers.
L'archive ouverte pluridisciplinaire HAL, est destinée au dépôt et à la diffusion de documents scientifiques de niveau recherche, publiés ou non, émanant des établissements d'enseignement et de recherche français ou étrangers, des laboratoires publics ou privés. 


\title{
A Data Driven Temperature Compensation Approach for Structural Health Monitoring using Lamb Waves
}

\author{
C. Fendzi ${ }^{1}$, M. Rébillat ${ }^{1}$, N. Mechbal ${ }^{1}$, M. Guskov ${ }^{1}$, and G. Coffignal ${ }^{1}$ \\ ${ }^{1}$ PIMM Laboratory, UMR CNRS 8006 Arts et Métiers ParisTech, \\ 151 Boulevard de l'Hôpital 75013, Paris, France \\ claude-marcellin.fendzi-tefoit@ensam.eu
}

\begin{abstract}
This paper presents a temperature compensation method for Lamb wave structural health monitoring. The proposed approach considers a representation of the piezo-sensor signal through its Hilbert transform that allows one to extract the amplitude factor and the phaseshift in signals caused by temperature changes. An ordinary least square (OLS) algorithm is used to estimate these unknown parameters. After estimating these parameters at each temperature in the operating range, linear functional relationships between the temperature and the estimated parameters are derived using the least squares method. A temperature compensation model is developed based on this linear relationship that allows one to reconstruct sensor signals at any arbitrary temperature. The proposed approach is validated numerically and experimentally for an anisotropic composite plate at different temperatures ranging from $16^{\circ} \mathrm{C}$ to $85^{\circ} \mathrm{C}$. A close match is found between the measured signals and the reconstructed ones. This approach is interesting as it needs only a limited set of piezo-sensor signals at different temperatures for model training and temperature compensation at any arbitrary temperature. Damage localization results after temperature compensation demonstrate its robustness and effectiveness.
\end{abstract}

Keywords: Structural health monitoring, temperature compensation model, Lamb waves, anisotropic composite materials, ordinary least squares.

\section{Introduction}

The fundamental challenges in the field of Structural Health Monitoring (SHM) are damage detection and localization (Sohn et al., 2003; Su and Ye, 2009). In guided wave structural health monitoring (GWSHM), a transducer network is mounted on a structure and is used to record wave signals in order to extract some damage related information (Farrar et al., 1999). Considering the widely used pitch-catch technique, one of the transducers is used as an actuator to excite the structure and the others are used as sensors to measure Lamb wave signals. The damage detection is performed by subtracting the signals captured from the structure in a healthy state (without damage) from those captured in a current unknown state (Ihn and Chang, 2008; Lu et al., 2006; Michaels, 2008; Moll et al., 2010). The difference of signals is used to locate and size the damage in the structure. This simple but often effective technique for damage detection can become a challenging task when environment or operational conditions (EOC) change (Andrews et al., 2008; Lee et al., 2011). Temperature change is the dominant EOC property that affects the robustness 
of a GWSHM system. Temperature changes often cause changes in instantaneous amplitude and instantaneous phase of a signal, thus signal stretching can be observed. It has been shown that, in the presence of changing temperature, simple subtraction of a single baseline signal from the current signal is not sufficient to discriminate between changes due to damage and those due to temperature variation. In order to mitigate this problem, many researchers have conducted theoretical and experimental investigations on the change in guided waves caused by temperature fluctuations (Kijanka et al., 2013; Konstantinidis et al., 2006; Lee et al., 2011; Lu and Michaels, 2005). Futhermore, the SHM research community has focused on developing techniques for temperature compensation in GWSHM damage detection. The most widely used techniques include optimal baseline selection (OBS) and baseline signal stretch (BSS). The OBS temperature compensation technique is a data-driven approach in which a collection of baseline signals are measured over a temperature range, and a specific criterion is used to select the best matched baseline signal for damage detection (Konstantinidis et al., 2006; Lu and Michaels, 2005). Commonly used criteria include least squares error methods or maximum residual amplitude of the signal after subtraction(Clarke et al., 2010). This compensation technique requires a very large amount of baseline signals as well as good temperature resolution in the signal acquisition to obtain a sufficiently low post-subtraction noise level. In practical applications these important requirements are not always available. Unlike the OBS, the BSS compensation techniques seek to build a model of the effects of temperature change on wave signals (Harley and Moura, 2012; Michaels and Michaels, 2005). The advantage of this technique is that it requires few baseline signals. For example Michaels and Michaels (2005) proposed a BSS compensation strategy based on time-stretch estimation using local temporal coherence, which can be seen as a measure of the time-dependent shape change between two signals. They conducted experiments on an aluminum plate and showed the effectiveness of their approach in discriminating structural damage from temperature change. Clarke et al. (2010) proposed to combine both OBS and BSS schemes to reduce the amount of baseline data needed for the temperature compensation. Recently, Roy et al. (2014) proposed a physics-based approach for temperature compensation of piezoelectric sensor signals, which takes into account the influence of temperature on physical properties of the structure under study as well as the piezo-transducer and adhesive interface. They validated their approach experimentally on stiffened panels made of aluminum alloy and showed the effectiveness of the methodology to detect structural damages. This approach requires explicit temperature dependency of material properties of the base plate, piezo-transducer and adhesive interface. Furthermore the approach needs training with prior data which are not always available. Wang et al. (2014) presented a temperature compensation method based on Adaptive Linear Neuron (ADLINE) for Lamb wave-based damage detection in composite plates. The main limitations of this method include the simplistic representation of the signal (single layer and 2 neural network weights) as well as the choice of the activation function. Furthermore, the gradient descent method used for estimating the unknowns parameters does not always warrant an optimal solution.

In this study, we propose a data driven compensation strategy for anisotropic composite materials. Since the temperature effect causes changes in piezo-sensor signals through an amplitude variation and a phase-shift, the approach considers an exact representation of the sensor signal through its Hilbert transform in order to extract these two parameters (amplitude and phaseshift). Afterward, the Levenberg-Marquardt algorithm (Lourakis, 2005; Roweis, 1996) is used to estimate these two parameters. The compensation methodology consists of two steps: first, the instantaneous amplitude and the instantaneous phase of the signals are extracted using the Hilbert transform, after we apply a parameter estimation approach to the temperature compensation 
problem using the measured piezo-sensor signals at two different temperatures, one considered as reference. Second, after estimating the unknown parameters at each temperature in the operating range, we build a regression model for these parameters using a least squares method. The signal reconstruction process at any temperature in the operating range can then be achieved using the regression model.

One of the major difficulties when considering numerical model validation of any active SHM approach that copes with temperature effects is to have available an updated model which accurately reproduces the effects of temperature on the composite and on the behavior of glued active elements (sensors and actuators). Thereby, in a first stage, we have chosen to apply our method directly on an actual laboratory anisotropic composite, with bonded piezoelectric elements, that has been exposed to different temperatures. The experimentation allows us to handle the overall behavior in temperature of the composite. Afterwards in a second stage, we have built a numerical model that has realistic behavior with respect to temperature. This numerical model allows us to perform parametric studies and to open key discussions regarding the applicability of our approach to in-service structures. It is worth noting that the objective of the numerical simulation is to validate more precisely the proposed compensation method, not to be compared to the experimental results. Before comparison, an updated model has to be constructed. It is not the framework of this study.

\section{Proposed approach for temperature compensation}

\subsection{Model}

Temperature-related effects that affect guided wave-based SHM system performance are usually related to temperature dependence of the transducers' performance as well as mechanical properties of the structure under study (Raghavan and Cesnik, 2008). These effects are usually observed as a variation in the instantaneous amplitude and phase of the signal, leading to a variation of the energy of the signal as well as a variation of the wave velocity or the travel time. As an example, Figure 1 illustrates the effect of temperature on the wave signal. An amplitude factor and a phase-shift between these two signals can be observed.

Considering these aspects, it can be assumed that the temperature effect on a guided wave signal $x(t)$ can be represented as:

$$
x(t) \stackrel{\text { Temperature effect }}{\longrightarrow} A(t, T) x\left(t-t_{r}(t, T)\right)
$$

where $A(t, T)$ is an instantaneous amplitude factor at temperature $T$, and $t_{r}(t, T)$ is a modified travel time seen as a time delay. Assuming constant values for $t_{r}(t, T)$ and $A(t, T)$ in a specific time window, the effect of the time delay causes an overall phase-shift of the windowed-signal. Consequently, the time delay can be written as:

$$
t_{r}(t, T)=(1-\alpha(T)) t
$$

where $\alpha(T)$ is a stretch-time coefficient or scale factor (Harley and Moura, 2012). Based on these assumptions, the temperature effect in the signal can be simplified as:

$$
x(t) \stackrel{\text { Temperature effect }}{\longrightarrow} A(T) x(\alpha(T) t)
$$

This shift modifies the instantaneous phase of the signal, then the stretch-time coefficient can be extracted using the Hilbert transform. 


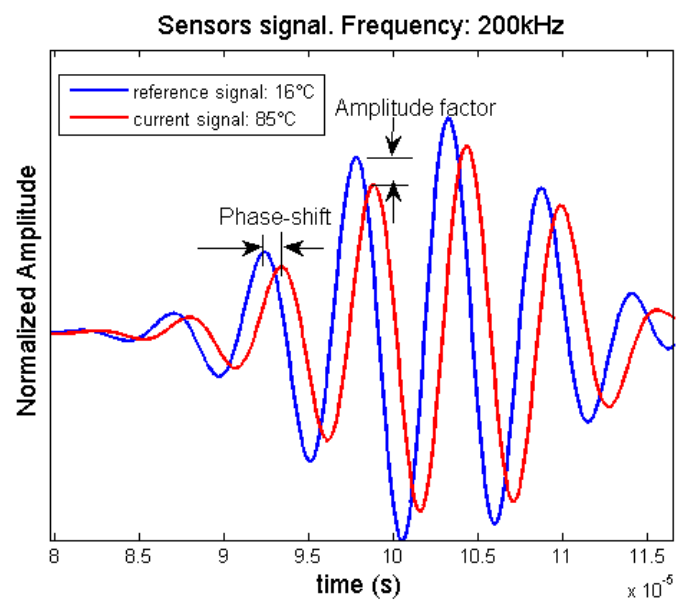

Figure 1: Illustration of the temperature effect on Lamb wave signal: experimental measurement in composite plate. The amplitude of the signal is normalized by scaling between 0 and 1 . This normalization is adopted in all the sequel.

\subsection{Hilbert transform for parameters extraction}

For any real signal $x(t)$, the corresponding analytic (complex) signal $z(t)$ is obtained as:

$$
z(t)=x(t)+j \mathcal{H}\{x\}(t)=\widehat{x}(t) e^{j \phi(t)}
$$

where $\mathcal{H}\{x\}(t)$ is the Hilbert transform of the real signal. $\widehat{x}(t)$ and $\phi(t)$ are the amplitude envelope and instantaneous phase of the signal $x(t)$, and are defined as:

$$
\widehat{x}(t)=\sqrt{x^{2}(t)+\mathcal{H}\{x\}^{2}(t)}, \quad \phi(t)=\arctan \left[\frac{\mathcal{H}\{x\}(t)}{x(t)}\right] .
$$

Considering two signals $x_{B}(t)$ (baseline signal) and $x_{T}(t)$ (current signal) at different temperatures $T_{B}$ and $T$, if the relation (3) is satisfied for these two signals, the effect of the temperature can be compensated by applying an amplitude factor and a phase-shift to the current signal. The analytic representation of the current signal is related to that of the baseline signal by:

$$
z_{T}(t)=A(T) z_{B}(t) e^{j\left(\phi_{B}(t)-\phi_{T}(t)\right)}
$$

Assuming the instantaneous phases at reference temperature $\left(T_{B}\right) \phi_{B}(t)$ and at any temperature $(T) \phi_{T}(t)$ are constant in a predefined time window with fixed length, Equation (6) can be simplified as:

$$
z_{T}(t)=A(T) z_{B}(t) e^{j \varphi(T)}
$$

where $\varphi(T)=\phi_{B}-\phi_{T}$. After amplitude correction and phase alignment from Equation(7), the real signal $x_{T}(t)$ can be reconstructed from its analytic form as:

$$
x_{T}(t)=\operatorname{Re}\left(z_{T}(t)\right)
$$

where $\operatorname{Re}(\cdot)$ is the real part.

Thus the unknown parameters vector in this temperature compensation approach is $\boldsymbol{\theta}=[A, \varphi]^{\mathrm{T}}$. The temperature compensation is then formulated as a parameter identification problem. 


\subsection{Parameter estimation}

In this section, we present the approach for estimating the unknown parameters vector $\boldsymbol{\theta}=[A, \varphi]^{\mathrm{T}}$. The solution which minimizes the sum of squarre error between the experimental signal and the model-reconstructed signal is given by:

$$
\boldsymbol{\theta}_{\min }=\underset{\boldsymbol{\theta} \in \mathbb{R}^{2}}{\arg \min } J(\boldsymbol{S}, \boldsymbol{\theta})
$$

where

$$
J(\boldsymbol{S}, \boldsymbol{\theta})=\sum_{i=1}^{N}\left[x_{T}\left(t_{i}\right)-x_{e}\left(t_{i} ; \boldsymbol{\theta}\right)\right]^{2}
$$

$x_{T}\left(t_{i}\right)(i=1, \cdots N)$ is a current signal sample measured at temperature $T$ and $x_{e}\left(t_{i} ; \boldsymbol{\theta}\right)$ is the estimated signal sample at the same temperature using the model described by Equations(7) and (8). $N$ is the number of samples in the considered time window. The solution to Eq (9) is obtained using the Levenberg-Marquardt algorithm (Lourakis, 2005; Roweis, 1996).

\subsection{Least squares regression model}

The regression model for temperature compensation is built using a least squares method (Montgomery et al., 2012). This method allows for estimation of the parameters of the regression function driving the model. The regression model is given by the equation:

$$
y_{i}=f\left(\boldsymbol{P}, T_{i}\right)+\text { error }
$$

where $y_{i}(i \in\{1, M\})$ are the estimated parameters $(A$ and $\varphi)$ at each temperature $T_{i} . f\left(\boldsymbol{P}, T_{i}\right)$ is the regression function which depends on the temperature $T_{i}$ and a vector of parameters $\boldsymbol{P}$. The vector of parameters $\boldsymbol{P}$ is estimated by minimizing the sum of squared error:

$$
\mathrm{SSE}=\sum_{i=1}^{M}\left(y_{i}-f\left(\boldsymbol{P}, T_{i}\right)\right)^{2},
$$

The shape of the regression function depends on the trend of the training points.

\section{Temperature compensation methodology and signal reconstruction process}

Figure 2 shows the procedure for the temperature compensation and signal reconstruction at any temperature. The overall process is described by the following sequential 6 steps:

Step 1 Collect a set of wave signals $\left\{\boldsymbol{S}_{\text {reference }}, \boldsymbol{S}_{1}, \cdots, \boldsymbol{S}_{M}\right\}$ at $M+1$ different temperatures $\left\{T_{\text {reference }}, T_{1}, \cdots, T_{M}\right\}$ from the structure under study. The value of $M$ should depend on the temperature range and the temperature increment.

Step 2 Consider two signals, the baseline signal $\boldsymbol{S}_{\text {reference }}$ and a current signal $\boldsymbol{S}_{q}(q \in\{1, \cdots, M\})$ at temperature $T_{q}$, and isolate the first wave packet using an appropriate time window. More than one wave packet can also be considered by increasing the length of the time window.

Step 3 Estimate the amplitude factor $A\left(T_{q}\right)$ and the phase-shift $\varphi\left(T_{q}\right)$ between these two signals. The estimation is performed using the Levenberg-Marquardt algorithm. The estimated parameters are used for model training. 
Step 4 Repeat Steps 2 and 3 for all the $M$ couples of signals $\left\{\boldsymbol{S}_{\text {reference }}, \boldsymbol{S}_{q}\right\}, q \in\{1, \cdots, M\}$ in the data set.

Step 5 Find a regression model with respect to the temperature for the estimated amplitude factor $A(T)$ and phase-shift $\varphi(T)$ using the set of the estimated amplitude $A\left(T_{q}\right)$ and phase-shift $\varphi\left(T_{q}\right)$ for each temperature value.

Step 6 Reconstruct a signal at temperature $T$, in the operating range using the regression model found in Step 5 (to estimate $A(T)$ and $\varphi(T)$ ) and the reference signal $x_{B}(t)$, via model given by Equations (7) and (8).

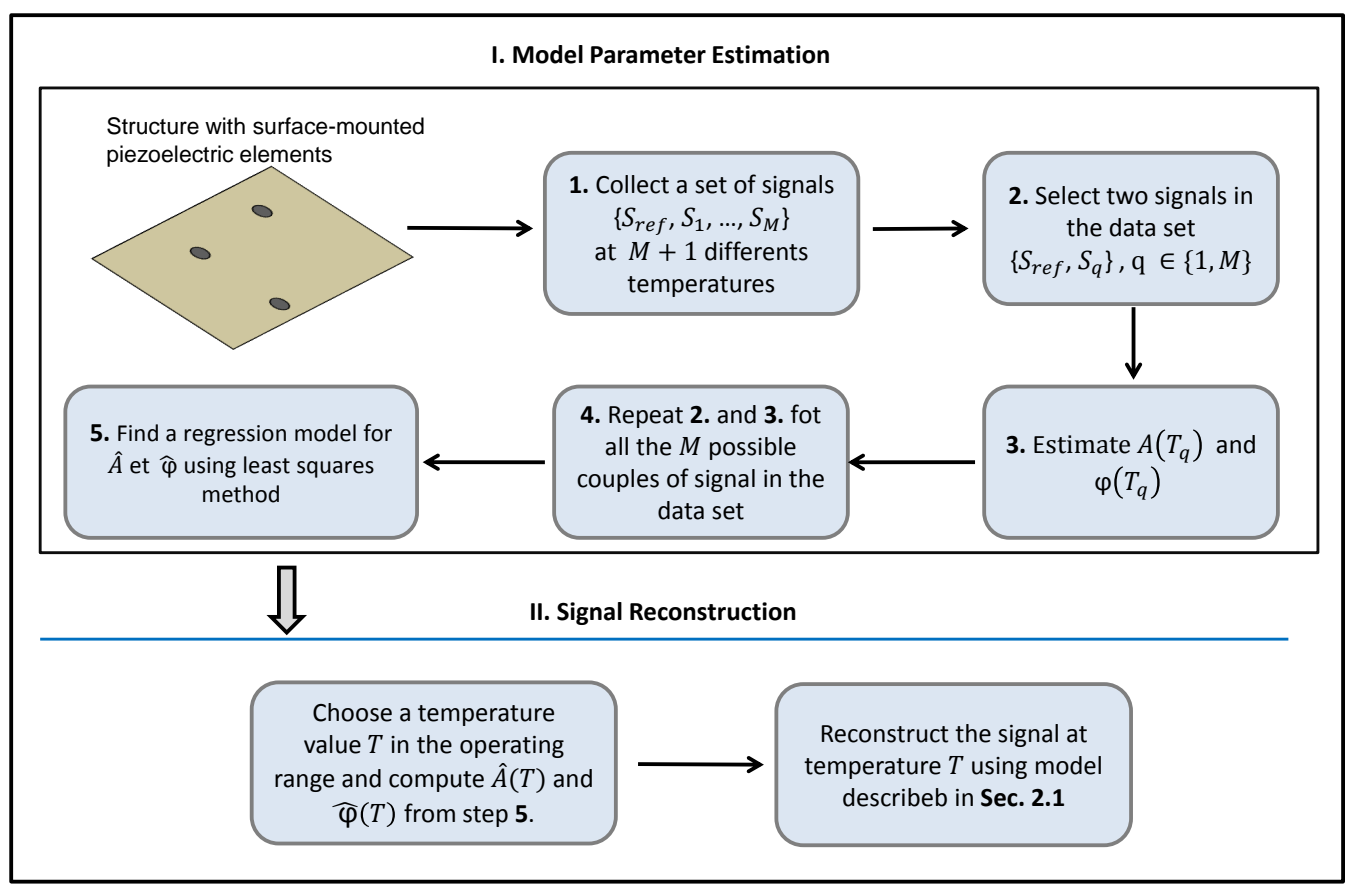

Figure 2: Proposed approach for temperature compensation and signal reconstruction

In order to compare the similarity of two time-domain signals, the normalized maximum residual amplitude (MaxError) is computed using the residual signal as:

$$
\text { MaxError }=20 \log \left[\frac{\max \left|x_{\text {reconstructed }}(t, T)-x_{\text {measured }}(t, T)\right|}{\max \left|x_{\text {measured }}(t, T)\right|}\right]
$$

where $x_{\text {reconstructed }}(t, T)$ and $x_{\text {measured }}(t, T)$ are the reconstructed signal and the experimentally measured signal at temperature $T$ respectively. Low values of MaxError in Equation (12) indicate good agreement between the experimental and reconstructed signals. The above described procedure for temperature compensation assumes that all the $M$ recorded signals can meet the compensation standard given by Equation (12). If it is not the case, the temperature increment should be adjusted and the procedure has to be repeated until a compensation standard is met. 
It is worth noting that the above methodology for temperature compensation assumes that the temperature field is constant along each actuator-sensor path.

\section{Experimental validation on composite laminates}

\subsection{Description of the experimental setup}

The test specimen is a four-ply CFRP composite laminate with stacking $\left[0^{\circ} /-45^{\circ} / 45^{\circ} / 0^{\circ}\right]$. The dimensions of each laminate are $400 \mathrm{~mm} \times 300 \mathrm{~mm} \times 0.28 \mathrm{~mm}$. Mechanical properties of the lamina are listed in Table 1.

Table 1: Mechanical properties of the lamina

\begin{tabular}{ccccccc}
\hline $\begin{array}{c}\text { Density } \\
\left(\mathrm{kg} / \mathrm{m}^{3}\right)\end{array}$ & $\begin{array}{c}\text { ply thickness } \\
(\mathrm{mm})\end{array}$ & $\begin{array}{c}E_{11} \\
(\mathrm{GPa})\end{array}$ & $\begin{array}{c}E_{22} \\
(\mathrm{GPa})\end{array}$ & $\begin{array}{c}E_{33} \\
(\mathrm{GPa})\end{array}$ & $\begin{array}{c}G_{12}=G_{13}=G_{23} \\
(\mathrm{GPa})\end{array}$ & $\nu_{12}$ \\
\hline 1554 & 0.28 & 69 & 69 & 8.1 & 4.8 & 0.03 \\
\hline
\end{tabular}

A set of $N=5$ piezoelectric (PZT) elements (Noliac NCE51) from NOLIAC Inc. are surfacemounted on the composite plate. Each piezoelectric element is $20 \mathrm{~mm}$ in diameter and $0.1 \mathrm{~mm}$ in thickness. The pitch-catch principle is considered here, wherein one PZT acts as an actuator while the others act as sensors. An illustration of the plate and sensor placement is shown in Figure 3. The coordinates of the piezoelectric elements are listed in Table 2.

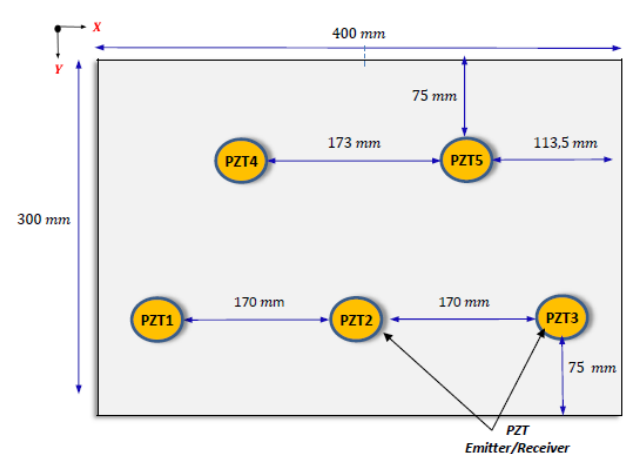

(a)

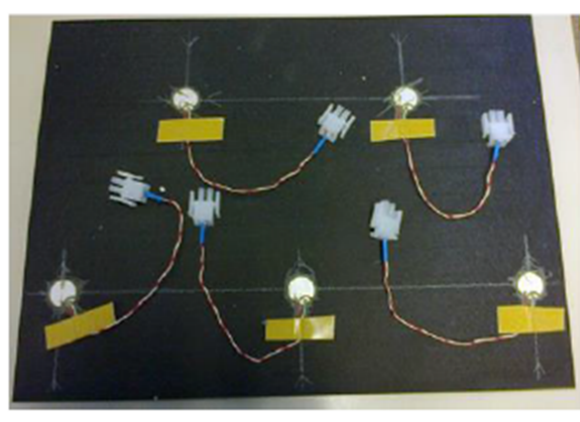

(b)

Figure 3: Sensors network with 5 piezoelectric patches mounted on the composite plate. (a) schematic, (b) experimental specimen.

Table 2: Coordinates of piezoelectric elements (center)

\begin{tabular}{cccccc}
\hline Transducers & $\mathrm{PZT}_{1}$ & $\mathrm{PZT}_{2}$ & $\mathrm{PZT}_{3}$ & $\mathrm{PZT}_{4}$ & $\mathrm{PZT}_{5}$ \\
\hline $\mathrm{x}(\mathrm{mm})$ & 30 & 200 & 370 & 113.5 & 286.5 \\
\hline $\mathrm{y}(\mathrm{mm})$ & 75 & 75 & 75 & 225 & 225 \\
\hline
\end{tabular}

Piezoelectric elements are actuated with a five-cycle sinusoidal tone burst at a central frequency of $f_{0}$, which is modulated by a Hanning window.

In the following, the excitation frequency is set to $f_{0}=200 \mathrm{kHz}$, and the signal reconstruction results after temperature compensation are only presented when PZT 5 acts as an actuator, the others acting as sensors. 
The excitation signal is generated by a 33500B series Waveform Generator, and amplified to $10 \mathrm{~V}$ using a voltage amplifier from FLC Electronics. The sensor measurements are visualized and recorded by a Tektronix Digital Phosphor Oscilloscope (DPO3014 series) whose sampling rate is set at $25 \mathrm{MHz}$. Figure 4(a) shows the experimental setup used to collect signals at ambient temperature $\left(T_{r e f}=16^{\circ} \mathrm{C}\right)$, taken as reference. After recording signals at ambient temperature, the composite plate was placed into an oven and signals were recorded at four $(M=4)$ different temperatures $\left\{38^{\circ} \mathrm{C}, 53^{\circ} \mathrm{C}, 69^{\circ} \mathrm{C}, 85^{\circ} \mathrm{C}\right\}$. A thermocouple placed on a specimen, which had the same mechanical properties as the composite plate, was used for temperature recording.

Figure 4(b) shows the signals recorded at PZTs 1,2,3 and 4 when PZT 5 is used as actuator for two different values of temperatures $\left(16^{\circ} \mathrm{C}\right.$ and $\left.53^{\circ} \mathrm{C}\right)$. As expected, it can be observed that the instantaneous amplitude and phase-shift are modified when the temperature changes.

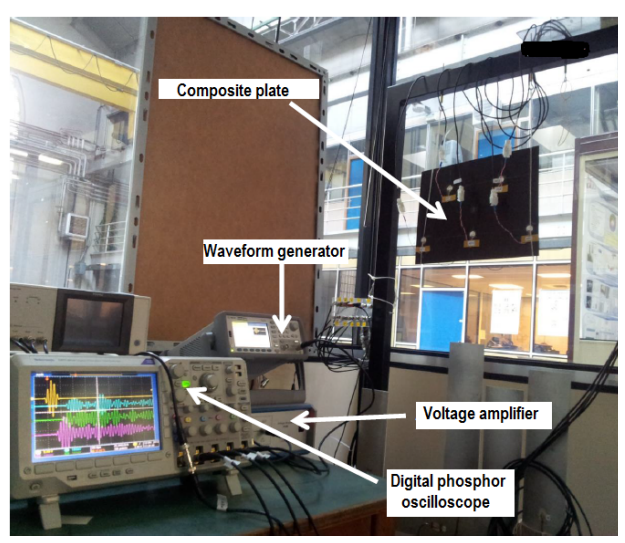

(a)

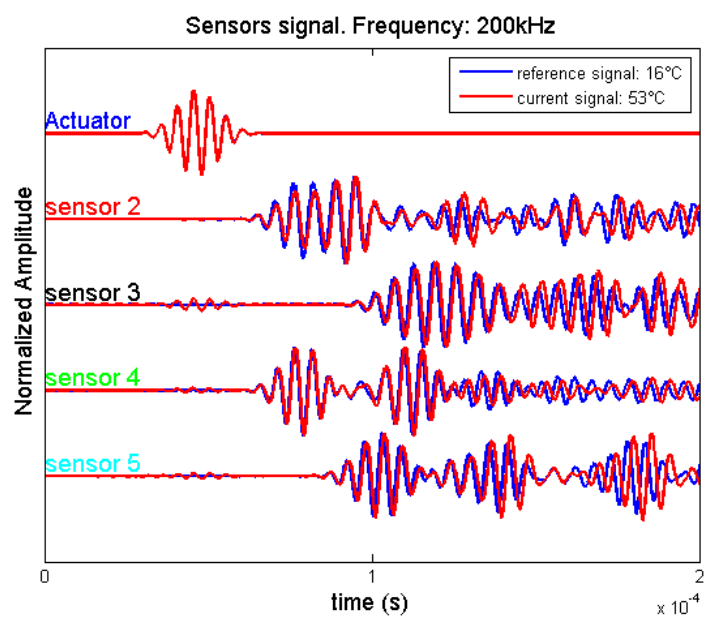

(b)

Figure 4: (a) Experimental setup at ambient temperature. (b) Sensors signals at 2 different temperatures

\subsection{Amplitude factor and phase-shift estimation}

As described in Section 3, the change in amplitude and phase-shift is estimated using the LevenbergMarquardt algorithm. In order to neglect boundary reflections, the compensation strategy described in this study is only applied to the first wave packet, for which the duration is approximatively equal to that of the excitation signal (as shown by dashed black rectangles in Figure 4(b)), where it is assumed that the instantaneous amplitude factor and phase-shift are constant. The procedure is performed to estimate the constant amplitude factor $A$ and phase shift $\varphi$ considering signals at two different temperatures (reference at $16^{\circ} \mathrm{C}$ and current at $53^{\circ} \mathrm{C}$ ). The estimated values for $A$ and $\varphi$ are listed in Table 3, for sensors 1,2,3 and 4. PZT 5 is used as actuator.

Table 3: Estimated parameters for $A$ and $\varphi$ obtained using the LevenbergMarquardt algorithm. $\mathrm{T}=$ $53^{\circ} \mathrm{C}$

\begin{tabular}{ccccc}
\hline Parameters & Actuator-sensor 1 & Actuator-sensor 2 & Actuator-sensor 3 & Actuator-sensor 4 \\
\hline$A$ & 0.9933 & 1.1025 & 1.0942 & 0.9949 \\
\hline \hline$\varphi($ rad $)$ & 0.7122 & 0.4803 & 0.4739 & 0.4331 \\
\hline \hline
\end{tabular}


Time Responses sensor: 1

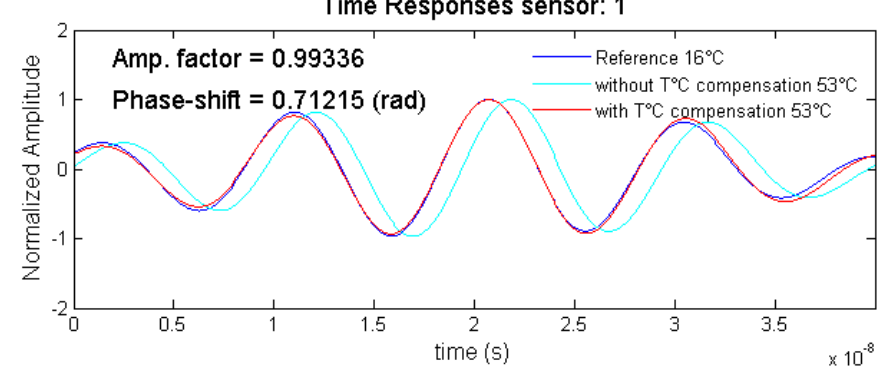

Difference of signals sensor: 1

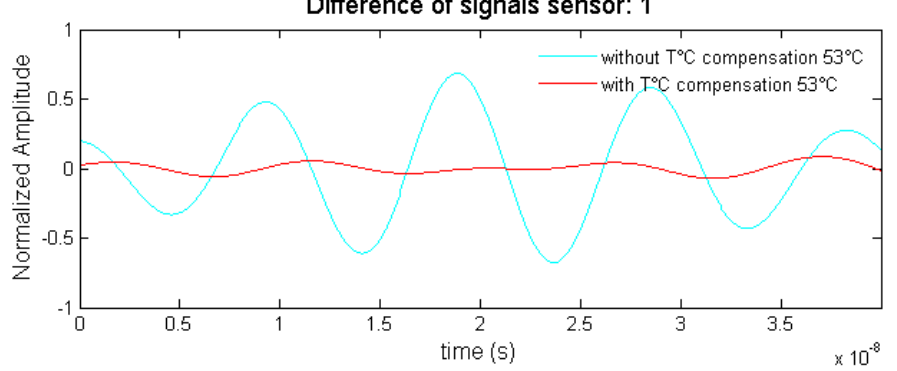

Figure 5: Signals with and without temperature compensation. (top) signal sensor 1, (bottom) difference of signal before and after compensation

Figure 5 shows both experimental and model-based reconstructed sensors signals at $53^{\circ} \mathrm{C}$ for sensor 1 when PZT 5 acts as actuator. The difference between the measured signals with and without temperature compensation is also presented. From this figure, a good degree of closeness between the experimental and reconstructed signals can be seen.

The procedure for parameter estimation is repeated for the other temperature values (as described in the temperature compensation procedure - Section 3) in order to find a model of variation of these parameters with respect to the temperature. Figures 6 and 7 show the variations of the amplitude factor $A$ and phase-shift $\varphi$ with respect to the temperature for sensors 1,2, 3 and 4 when PZT 5 is used as actuator. From the figures, a linear trend with the temperature for the amplitude factor and phase-shift can be observed. The regression function can then be written as: $f(\boldsymbol{P}, T)=a T+b$, where $\boldsymbol{P}=[a, b]^{T}$ has to be determined using least square method.

Using the value listed in Table 3 for PZT 5 at T $=53^{\circ} \mathrm{C}$, the parameters $\widehat{a}, \widehat{b}$ are estimated for each couple actuator-sensor and are listed in Table 4.

Table 4: Estimated parameters $\widehat{a}, \widehat{b}$ for PZT 5

\begin{tabular}{ccccc}
\hline Parameters & actuator-sensor 1 & actuator-sensor 2 & actuator-sensor 3 & actuator-sensor 4 \\
\hline$\widehat{a}_{A}\left({ }^{\circ} C^{-1}\right)$ & 0.0033 & 0.0001 & 0.0019 & -0.0005 \\
\hline$\widehat{b}_{A}$ & 0.8522 & 1.0965 & 1.0051 & 1.0119 \\
\hline$\widehat{a}_{\varphi}\left({ }^{\circ} C^{-1}\right)$ & 0.0173 & 0.0115 & 0.0118 & 0.0105 \\
\hline$\widehat{b}_{\varphi}$ & -0.2455 & -0.1549 & -0.1764 & -0.1458 \\
\hline
\end{tabular}

From the results listed in Table 4, we build linear regression fits as shown in Figures 6 and 7 (blue dashed lines). From these figures, a fair closeness can be seen between the training points and the least squares linear regression. It can also be observed that the amplitude factor has 
almost a linear trend with the temperature, while the phase-shift increases with the temperature. This trend can be roughly explained as, when the temperature increases, the Young's and shear moduli decrease, leading to a less stiff material. As a result, the speed of the Lamb waves decrease, which is equivalent to an increasing of the phase-shift. Moreover, temperature influence on piezo ceramics behavior also affect the Lamb wave propagation as pointed out by Roy et al. (2014) in a recent study.

After obtaining the regression model from training data, a sensor signal can be reconstructed at any specific temperature in the operating range, using only the reference signal and the compensation model (7).
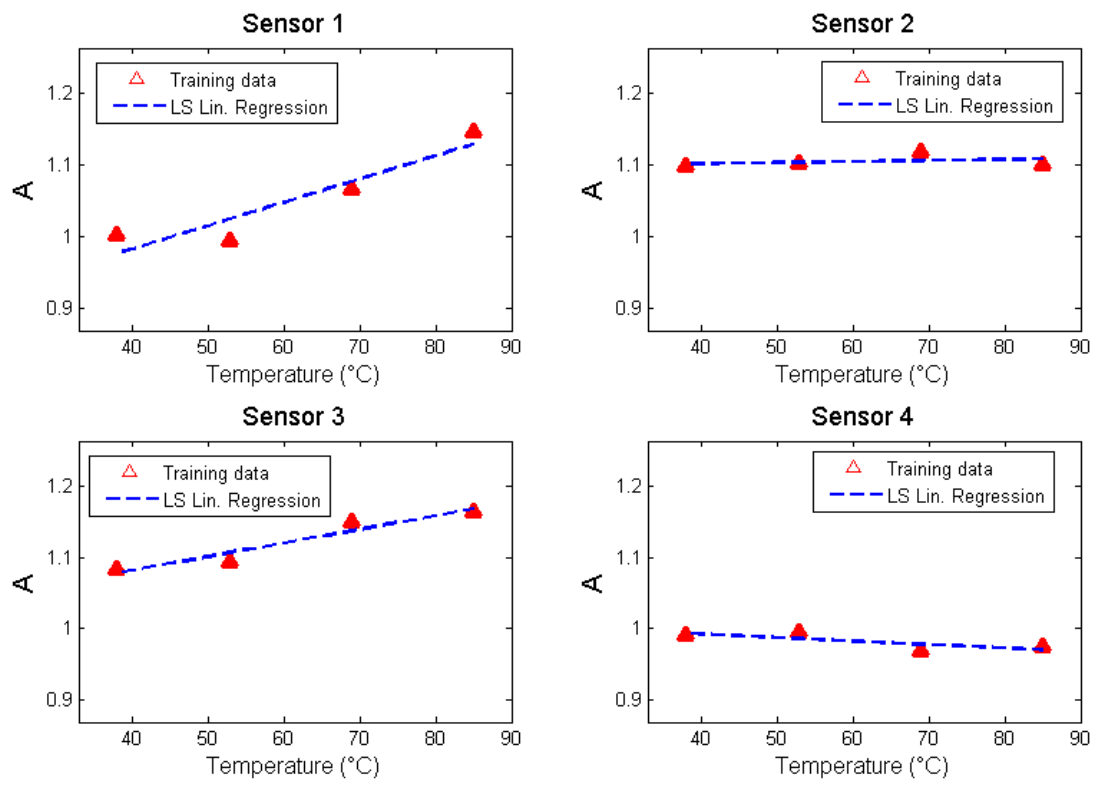

Figure 6: Amplitude factor A as a function of temperature (LS: least squares)

\subsection{Signal reconstruction and damage localization}

Figure 8 shows signal reconstruction errors in term of normalized maximum residual amplitude for different temperature values. Applying the compensation model to the sensor signals clearly reduces the maximum residual amplitude. Moreover, from low values of the normalized error after compensation, it can be seen that the compensated model is able to reconstruct any sensor signal with a reasonable accuracy for different temperatures in the operating range. Similar results are observed when the other PZTs are used as the actuator.

\section{- Estimation of the threshold for the normalized error}

The threshold of the normalized maximum residual amplitude from the baseline signal and modelreconstructed signal should be in an allowable range. For damage localization purposes, the acceptable value for the threshold should be related to the desired resolution of damage size in the structure. Roy et al. (2014) recently showed the effect of damage on the sensors signals recorded in an aluminum plate. Like temperature, structural damage causes variation in both instantaneous 

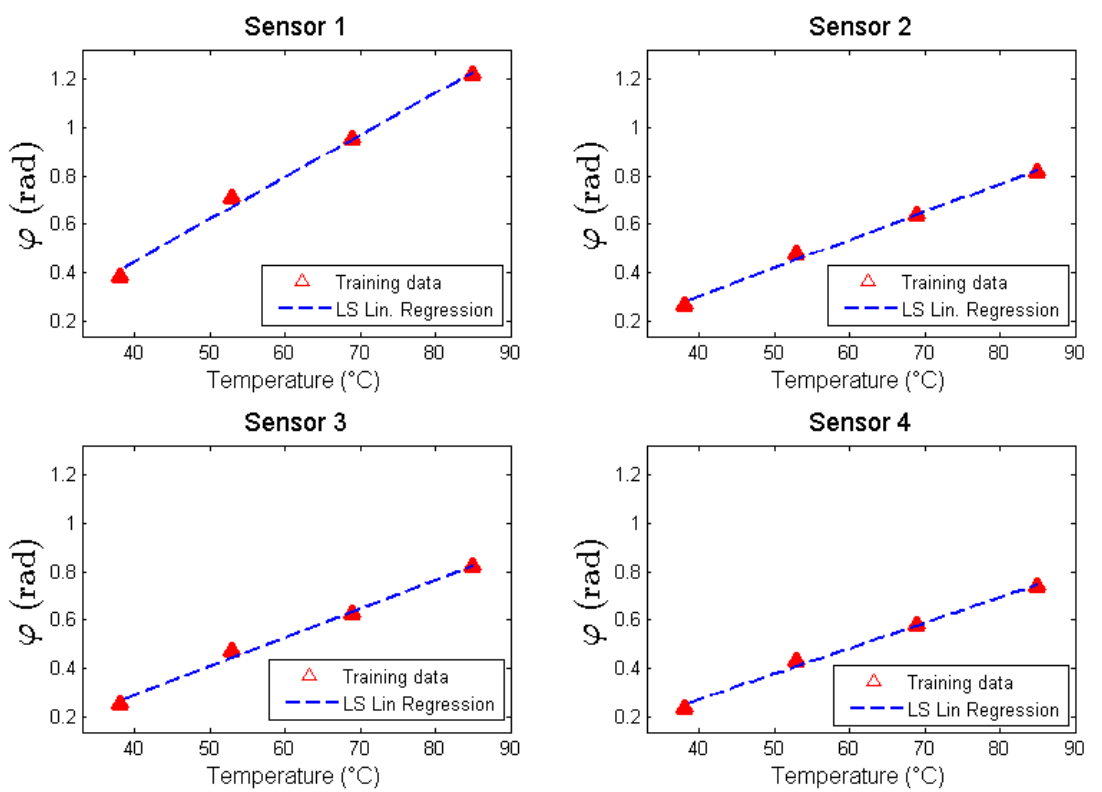

Figure 7: Phase-shift $\varphi$ as a function of temperature (LS: least squares)

amplitude and phase in the signals. With this in mind, the effect of the temperature must be distinguished from that of the damage. To do so, the effect of damage in the signals is evaluated with the same metric (Equation (12)) as for the temperature effect. This approach allows one to select an allowable threshold for the signal reconstruction error, with respect to the size of the damage which has to be localized. In order to estimate the lower limit of the normalized error after signal reconstruction, we introduce into the previous composite laminate a $14 \mathrm{~mm}$ damage impact as shown in Figure 9. The normalized error for this damaged configuration computed using Equation (12) is also shown in Figure 8 (green lines). In this damaged case, $x_{\text {reconstructed }}$ refers to the signal recorded in the damaged plate while $x_{\text {measured }}$ refers to the signal recorded in the healthy plate. The results show that applying the compensation procedure to the signals clearly reduces the post-subtraction noise level. From the maximum residual amplitude showed in Figure 8 , the allowable threshold to ensure detection of $14 \mathrm{~mm}$ impact damage is set at $-15 \mathrm{~dB}$. This threshold value is defined as $\max ($ MaxError $)$ from all sensors.

\section{- Damage localization}

The proposed temperature compensation approach is validated for damage localization in a composite laminate under a changing temperature environment. The above described $14 \mathrm{~mm}$ impact damage is considered here. The damage is located at $(300,150) \mathrm{mm}$. Before introducing any damage, baseline sensors signals are first recorded from the structure without damage and under different temperatures $\left\{16^{\circ} \mathrm{C}, 38^{\circ} \mathrm{C}, 53^{\circ} \mathrm{C}, 69^{\circ} \mathrm{C}, 85^{\circ} \mathrm{C}\right\}\left(16^{\circ} \mathrm{C}\right.$ is considered as the reference temperature). After creating impact damage on the composite plate, sensor signals are once again measured at the same temperatures as in the healthy case.

The damage localization algorithm used here is the standard delay-and-sum imaging approach (Fendzi et al., 2015; Ihn and Chang, 2008; Michaels, 2008) which uses the time-of-flight (ToF) 

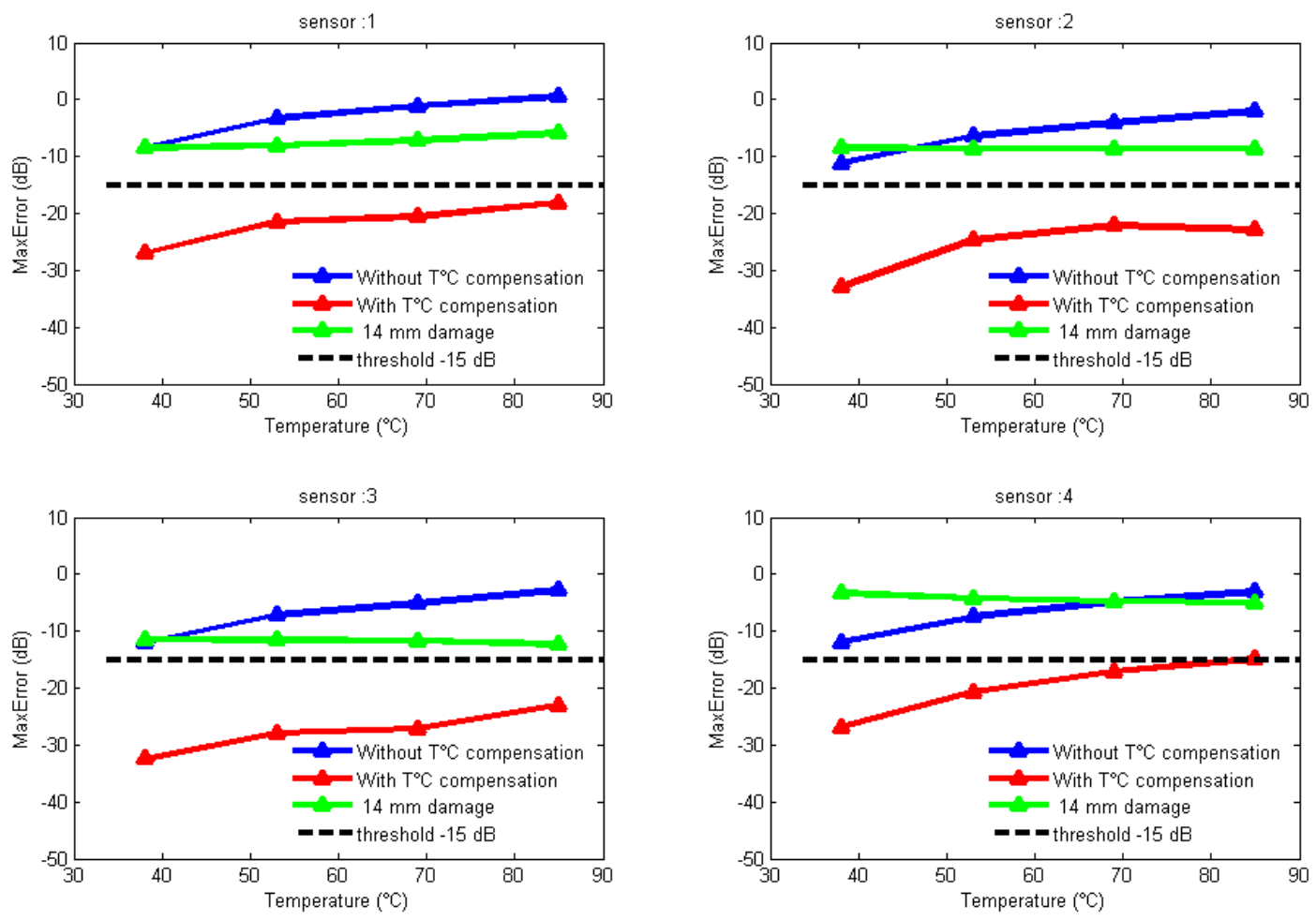

Figure 8: Normalized maximal error in $d B$ at different temperatures obtained using Equation (12) PZT 5 used as actuator - Experimental study

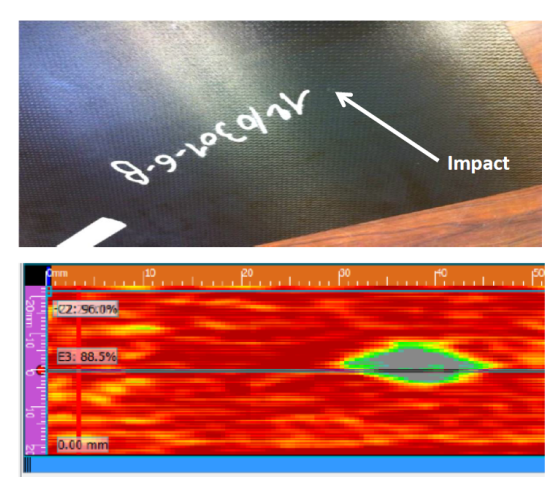

(a)

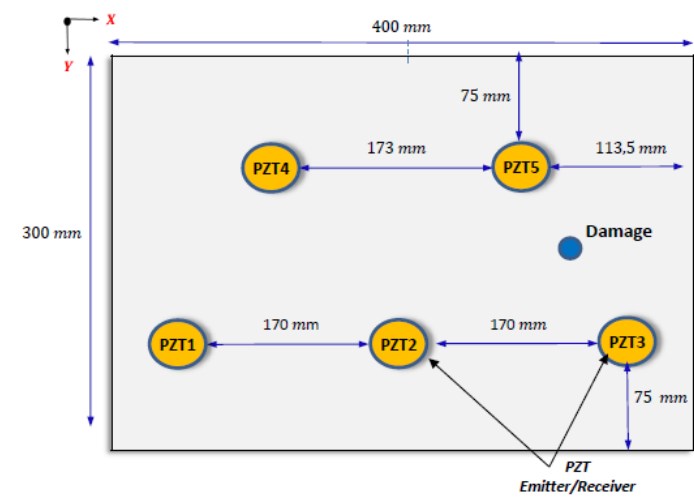

(b)

Figure 9: (a) Composite plate with the $14 \mathrm{~mm}$ impact (top) C-scan of the impact (bottom), (b) Coordinates of the impact $(300,150) \mathrm{mm}$.

of the signals scattered by damage, which are obtained from the difference between the sensors signals at the healthy state and the sensors signals recorded at the actual damaged state (under 
the same temperature). First, the wave signals from the healthy structure (at specific elevated temperature) are reconstructed using the reference (healthy) signal at $16^{\circ} \mathrm{C}$ and the compensation model parameters estimated for this specific temperature. Next, the damage localization procedure is applied for damage imaging. The damage imaging algorithm requires ToF information, which, for a single actuator-sensor $(a-s)$ path, can be expressed as:

$$
\mathrm{ToF}^{a-s}=\frac{\sqrt{\left(x_{d}-x_{a}\right)^{2}+\left(y_{d}-y_{a}\right)^{2}}}{V_{g}\left(T, \alpha_{a}\right)}+\frac{\sqrt{\left(x_{d}-x_{s}\right)^{2}+\left(y_{d}-y_{s}\right)^{2}}}{V_{g}\left(T, \alpha_{s}\right)}
$$

where $\left(x_{a}, y_{a}\right),\left(x_{s}, y_{s}\right),\left(x_{d}, y_{d}\right)$ are the actuator, sensor and damage coordinates respectively, and $V_{g}(T, \alpha)$ is the group velocity of the Lamb wave, which depends on the propagation direction in a composite material, $\alpha$, as well as on the temperature. $V_{g}\left(T, \alpha_{a}\right)$ and $V_{g}\left(T, \alpha_{s}\right)$ represent the group velocity in the actuator-damage path and damage-sensor path respectively. Figure 10 shows the experimental group velocity profile at different temperatures for the four-ply composite plate described earlier. For this frequency $(200 \mathrm{kHz})$, the mostly dominant Lamb mode is $\mathrm{S}_{0}$, with a quasi-isotropic group velocity profile whose nominal value is $5350 \mathrm{~m} / \mathrm{s}( \pm 2 \%)$.

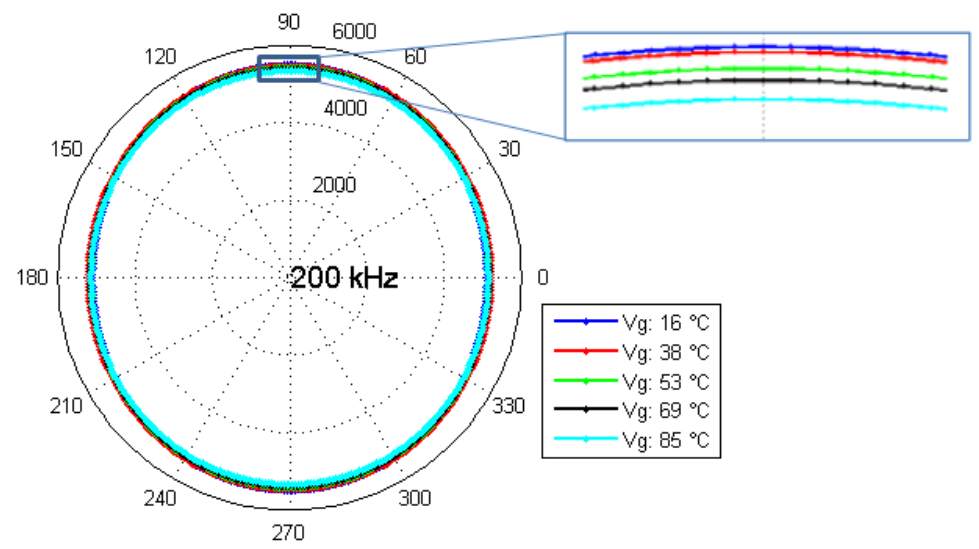

Figure 10: Group velocity profile at different temperatures: frequency $200 \mathrm{kHz}$

Figure 11 shows the damage imaging results before and after applying the compensation procedure at $38^{\circ} \mathrm{C}$. The distance between the estimated damage position and the centre of the true damage position at each temperature is also presented in Table 5. It can be clearly observed that using the temperature compensation model leads to an improved and accurate estimate of the damage location. Without compensation, the localization error becomes high with an ambiguous result for the damage position. One can also observe that the localization error increases with temperature. This trend is the consequence of the fact that the maximum residual error also increases with temperature, which is an unsurprising result. These results demonstrate the effectiveness and the applicability of the proposed temperature compensation scheme for damage localization.

\section{Numerical validation on composite laminate}

Numerical simulations were performed for the composite laminate described in Section 4.1. The material properties, the sensor placement and the dimensions of the plate remain the same. The $25^{\circ} \mathrm{C}-80^{\circ} \mathrm{C}$ temperature range in steps of $5^{\circ} \mathrm{C}$ is investigated numerically, $25^{\circ} \mathrm{C}$ taken as a reference temperature. The mechanical properties at elevated temperature are listed in Table 6 , where 


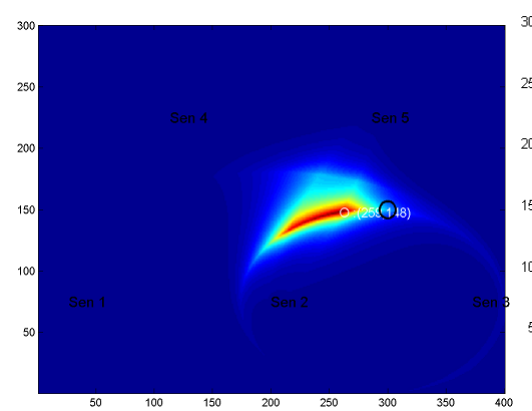

(a)

Without temperature compensation. Baseline at $16^{\circ} \mathrm{C}$ Damaged at $38^{\circ} \mathrm{C}$

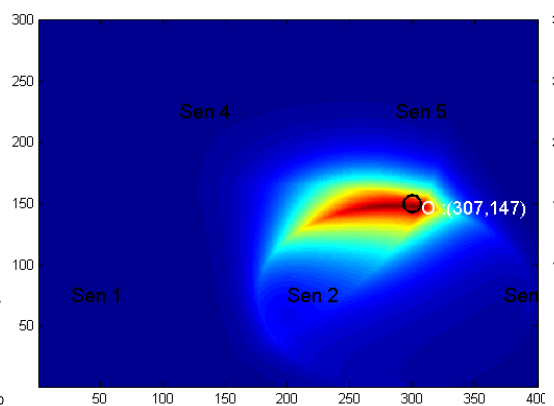

(b)

With temperature compensation. Reconstruction at $38^{\circ} \mathrm{C}$ Damaged at $38^{\circ} \mathrm{C}$

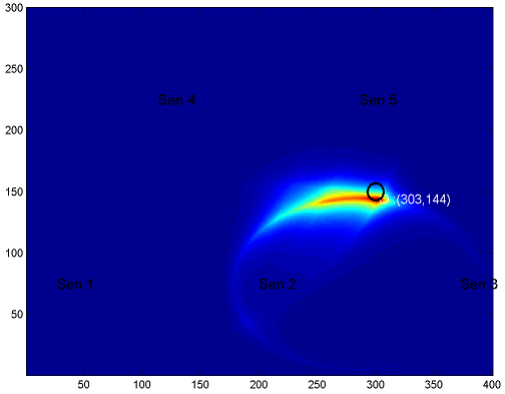

(c)

Baseline at $38^{\circ} \mathrm{C}$ Damaged at $38^{\circ} \mathrm{C}$

Figure 11: Damage imaging of the composite laminate at temperature $38^{\circ} \mathrm{C}$, black circle represents true damage location. Relevant dimensions of the plate is shown in Figure 3. The intensity values vary from 0 (blue color) to 1 (red color). The damage location is given by the highest value (the reddest zone).

Table 5: Localization error in $m m$ : distance between the estimated damage position and the centre of the true damage position at each temperature

\begin{tabular}{|l||c|c|c|c|}
\hline \multicolumn{1}{|c||}{ Temperature } & $38^{\circ} \mathrm{C}$ & $53^{\circ} \mathrm{C}$ & $69^{\circ} \mathrm{C}$ & $85^{\circ} \mathrm{C}$ \\
\hline \hline Wonfigurations & & & & \\
\hline Without Temperature Compensation & $41.04 \mathrm{~mm}$ & $100.84 \mathrm{~mm}$ & $68.62 \mathrm{~mm}$ & $102.31 \mathrm{~mm}$ \\
\hline Both signals at same Temperature & $6.71 \mathrm{~mm}$ & $7.21 \mathrm{~mm}$ & $10.77 \mathrm{~mm}$ & $11.18 \mathrm{~mm}$ \\
\hline
\end{tabular}

$\epsilon_{0}=8.854 \times 10^{12} \mathrm{~F} / \mathrm{m}$ is the vacuum permittivity. A linear relationship is used to obtain the corresponding values at intermediate temperatures.

It is worth noting that the objective of the numerical simulation is to validate more precisely the proposed compensation method. Indeed, measurements at only four temperatures are available experimentally which is not enough to validate the whole temperature compensation method. The numerical study has thus been conducted in order to be able to generate data points each $5^{\circ} \mathrm{C}$, and thus to really assess the validity and the robustness of the proposed method in the case of small temperature gradient. As the retained numerical model is a rather crude approximation of reality (lake of more values of mechanical properties at elevated temperature, no numerical model updating), the experimental and numerical results can not be directly compared in any way.

Table 6: Mechanical properties at elevated temperature

\begin{tabular}{|c||c||c||c||c||c||c|}
\hline Mech. properties & $E_{33}$ & $G_{12}$ & $G_{13}$ & $G_{23}$ & $E_{\text {glue }}$ & permittivity $\left(\epsilon_{r}\right)$ \\
& $(\mathrm{GPa})$ & $(\mathrm{GPa})$ & $(\mathrm{GPa})$ & $(\mathrm{GPa})$ & $(\mathrm{GPa})$ & \\
\hline $23{ }^{\circ} \mathrm{C}$ & 8.1 & 4.8 & 4.8 & 4.8 & 5.91 & $1930.9 \epsilon_{0}$ \\
\hline $120^{\circ} \mathrm{C}$ & 6.7 & 1.9 & 4.0 & 4.0 & 3.0 & $2639 \epsilon_{0}$ \\
\hline
\end{tabular}


The excitation signal is a five-cycle sinusoidal tone burst at a central frequency of $f_{0}=200 \mathrm{kHz}$, modulated by a Hanning window as in the experimental case.

Figure 12(a) shows the finite element model (FEM) of the composite plate with surface-mounted piezoelectric elements. The finite element package (Structural Dynamic Toolbox) used in this numerical study is a commercial toolbox developed for the Matlab environment. It is a software allowing one to perform finite element analysis, experimental modal analysis, and test/analysis correlation. Some details can be found at (Balmes and Deraemaeker, 2013). The numerical model was meshed with quadratic elements (4 nodes per element). The total number of degrees of freedom is 760,000 . The KirchhoffLove theory of plates has been used to model the plate, with the kinematic assumption that the thickness of the plate remains straight during wave propagation. This assumption seems reasonable since the wavelength in the plate at the frequency of $f_{0}=200 \mathrm{kHz}$ is about $22 \mathrm{~mm}$, which is much higher than the thickness of the plate $(1.12 \mathrm{~mm})$. The PZTs patches are modelled to reproduce the piezoelectric effect involved in experimental Lamb wave generation. For each PZT patch, 2 electrodes are designed. The Glue has also been modelled using 3D elements. The Glue's thickness is $0.1 \mathrm{~mm}$ and the PZT thickness is $1 \mathrm{~mm}$.

The transient analysis was achieved using an explicit Newmark scheme, with time step of $0.3 \mu s$. The number of points is equal to 2000, and the time interval of the simulation is [0 $0.6 \mathrm{~ms}$. Typical sensor responses at two different temperatures are shown in Figure 12(b) for PZTs 1, 2, 3 and 4 when PZT 5 acts as actuator.

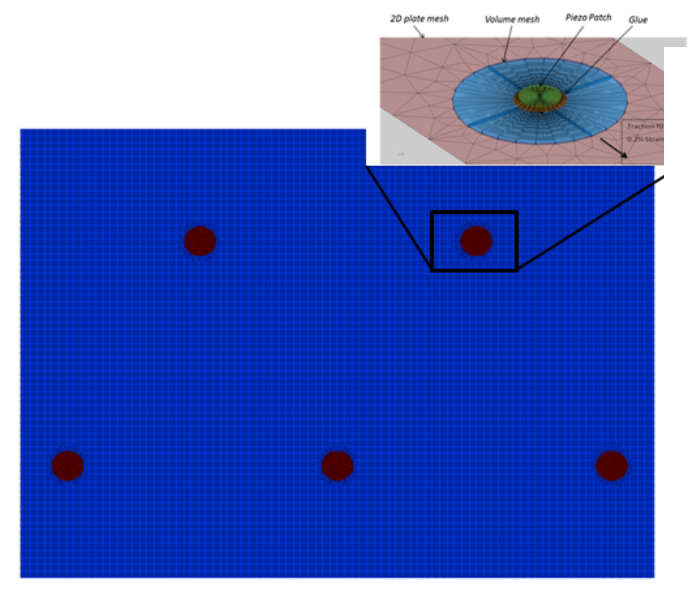

(a)

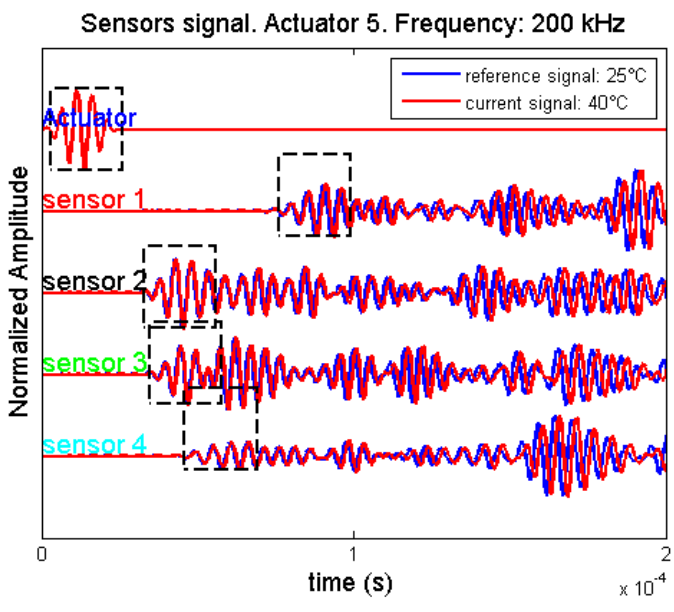

(b)

Figure 12: (a) FEM model with details of the piezoelectric element meshing (b) Sensor responses at temperatures $25^{\circ} \mathrm{C}$ and $40^{\circ} \mathrm{C}$.

\subsection{Parameters estimation and linear regression model}

The previously described procedure is used for estimating the amplitude factor and phase-shift at each temperature value in the operating range. The estimated values are listed in Table 7 when PZT 5 acts as actuator at $40^{\circ} \mathrm{C}$.

These estimations are performed at each temperature value and the linear model regression can 
Table 7: Estimated values for $A$ and $\varphi \cdot \mathrm{T}=40^{\circ} \mathrm{C}$

\begin{tabular}{ccccc}
\hline Parameters & Actuator-sensor 1 & Actuator-sensor 2 & Actuator-sensor 3 & Actuator-sensor 4 \\
\hline$A$ & 0.9218 & 0.9869 & 0.9755 & 0.9895 \\
\hline \hline$\varphi($ rad $)$ & 0.9613 & 0.3905 & 0.4987 & 0.6085 \\
\hline \hline
\end{tabular}

be built using the least squares method. The linear regression function and the training points for the amplitude factor and the phase-shift are plotted in Figures 13 and 14 when PZT 5 acts as the actuator.
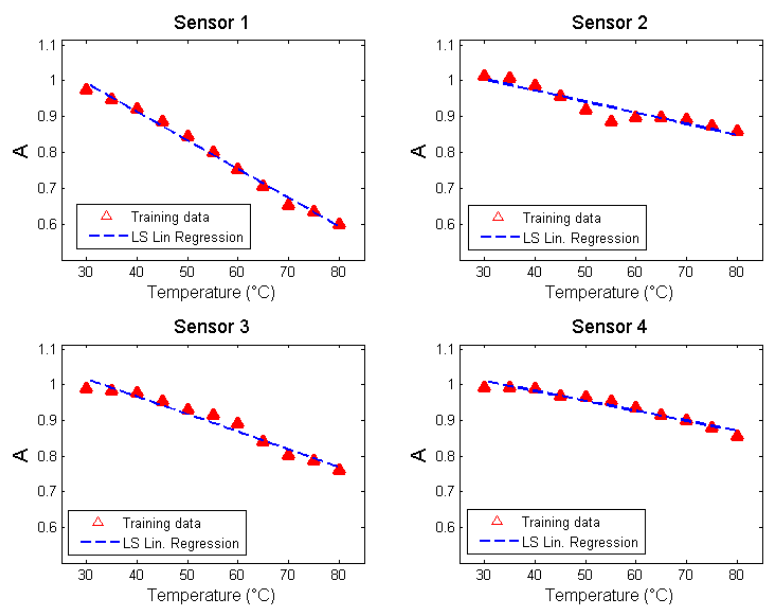

Figure 13: Amplitude factor A with the temperature (LS:least squares) - Numerical study
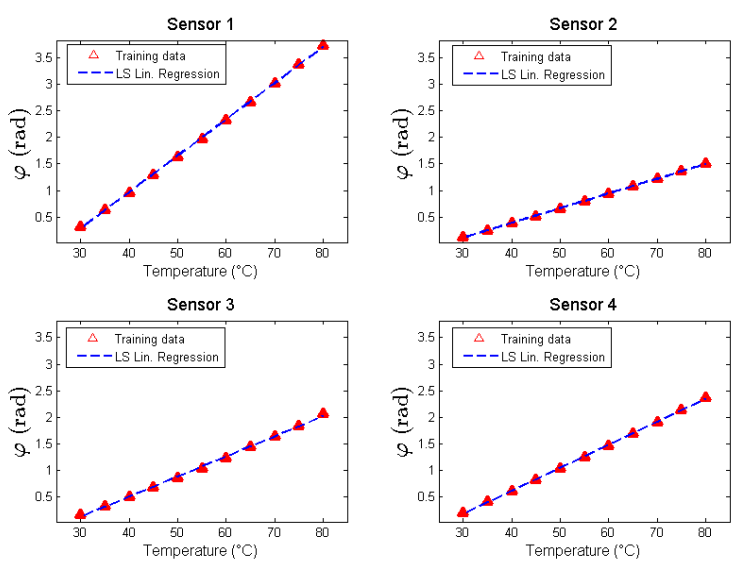

Figure 14: Phase-shift $\varphi$ with the temperature (LS: least squares) - Numerical study 


\subsection{Signal reconstruction and damage localization}

The signal reconstruction process can been achieved using the regression model and the signal at the reference temperature. Figure 15 shows the difference between the numerical and the modelbased reconstructed sensors signals at $40^{\circ} \mathrm{C}$ when PZT 5 acts as the actuator. A good match between these two signals can be seen.
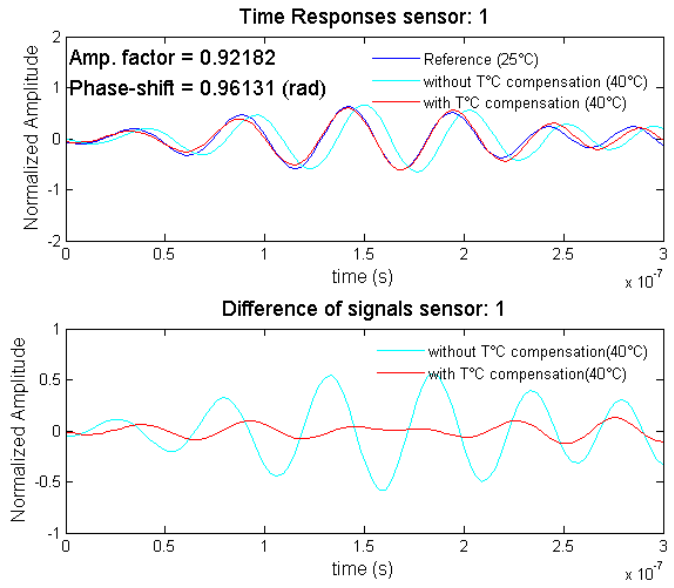

Figure 15: Signals with and without temperature compensation. (top) signal sensor 1, (bottom) difference of signal before and after compensation - Numerical study at $40^{\circ} \mathrm{C}$

The numerical validation of the temperature compensation model is performed for damage localization. The damage considered here is $20 \mathrm{~mm}$ diameter with coordinates $(200,150) \mathrm{mm}$. The damage is modeled by a local loss of stiffness of $95 \%$ (change in Young's and shear modulus of the material) (Chatterjee, 2001; Garnich and Akula, 2009). In this damaged case, the normalized maximal error can be computed using Equation (12). The results allow one to set the allowable threshold of the compensation to locate this damage size. The maximal residual error is computed using Equation (12) with temperature compensation, without temperature compensation and for the $20 \mathrm{~mm}$ damaged signal as in the experimental study, and the results are presented in Figure 16 for PZT 5 used as the actuator. The results show that applying the compensation model to the signals clearly reduces the post-subtraction noise level. Furthermore, the results show that the damage results and the uncompensated results in temperature have the same level of contribution in ErrorMax, which can increase the false positive rate in the damage detection process, if no compensation is applied.

As in the experimental study, the damage localization procedure is performed before and after temperature compensation to validate the proposed compensation model. Figure 17 shows the damage localization results. The black circle represents the true damage location while the red region represents the estimated damage location. Once more, the results show the capability of the model to compensate for temperature influences on the sensor signal in order to accurately locate structural damage.

\section{Discussion}

The proposed temperature compensation model has been validated in both experimental and numerical case studies. Fairly good agreement is observed between the reconstructed piezo-sensor 

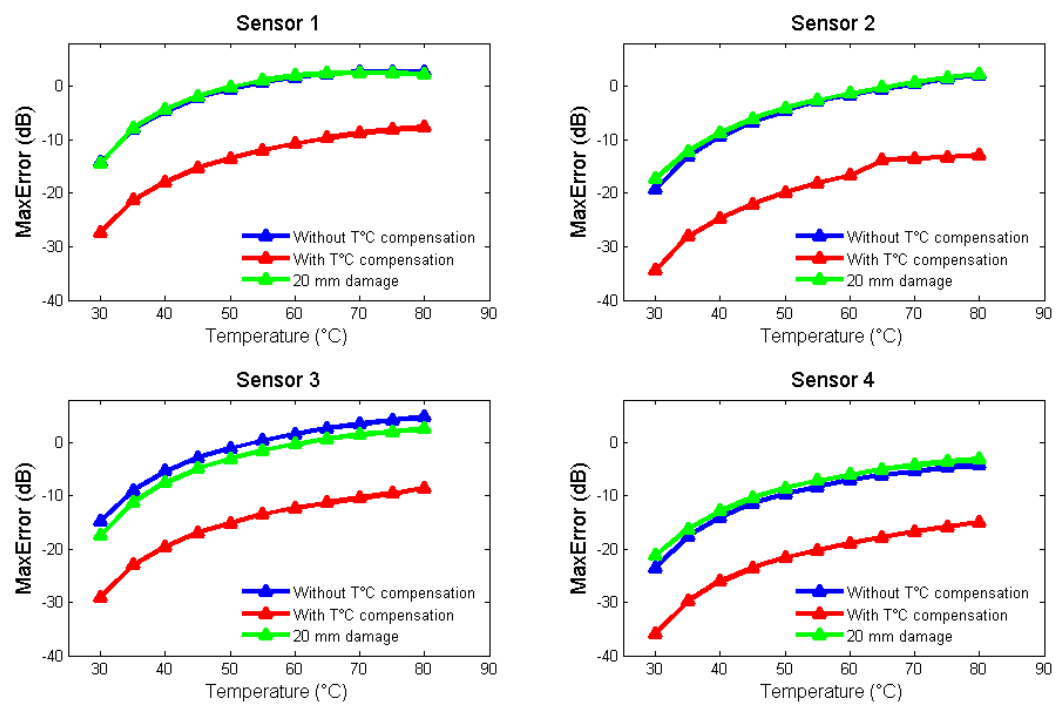

Figure 16: Normalized maximal error in $d B$ at different temperatures obtained using Equation (12) PZT 5 used as the actuator - Numerical study

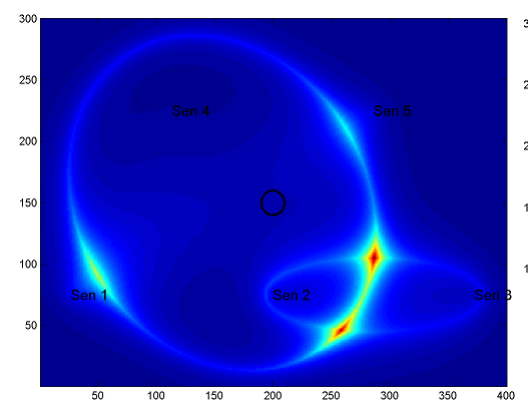

(a)

Without temperature

compensation.

Baseline at $25^{\circ} \mathrm{C}$

Damaged at $40^{\circ} \mathrm{C}$

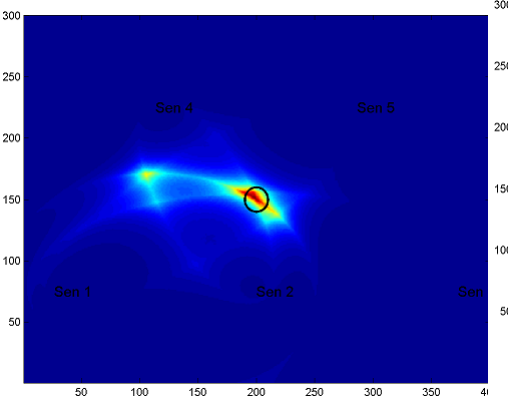

(b)

With temperature compensation.

Reconstruction at $40^{\circ} \mathrm{C} \quad$ Baseline at $40^{\circ} \mathrm{C}$

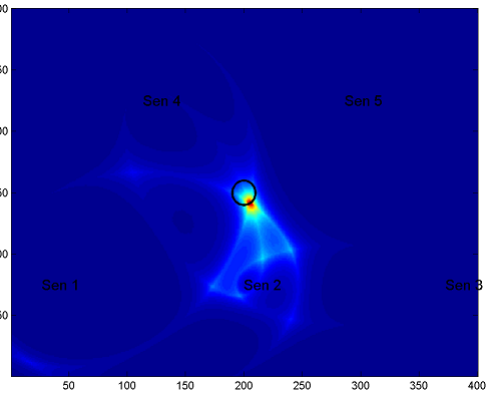

(c)

Damaged at $40^{\circ} \mathrm{C}$

Figure 17: Damage imaging of the composite laminate at $40^{\circ} \mathrm{C}$, black circle represents true damage location. Numerical study

signals and the experimental ones. The damage localization results after compensation show the performance of this approach. Although this algorithm is promising for SHM temperature compensation, certain points need further investigation before applying it to in-service structures.

- The temperature compensation parameters $(\mathrm{A}$ and $\varphi$ ) are estimated using a least squares approach. Since we assumed constant amplitude factor and phase-shift in the temporal window considered in the estimation, the compensation accuracy and the validity of our assumption would depend on the length of the time window considered in the estimation. In 
Figure 8 we noticed a surprising behavior for the normalized maximal error for sensor 2 at $85^{\circ} \mathrm{C}$. This behavior is probably due to the assumption of constant amplitude and phase-shift in the considered time window. Furthermore, it would not be surprising that the estimation becomes bad when the difference in temperature between the reference signal $\left(T_{\text {reference }}\right)$ and the current signal $(T)$ increases. Thus, further investigation should be performed about the right temperature resolution as well as the right time window which has to be considered. Furthermore, it could be interesting to consider the possibility of setting damage detection thresholds as a function of temperature, since the normalized error seems to increase with temperature changes, leading to a threshold value that might also change.

- The composite structure considered in these studies is quasi-isotropic, and it has been observed that the amplitude factor and phase-shift for this material exhibit a linear trend with temperature. It might be interesting of considering a more anisotropic layups where wave speed vary significantly with orientation, in order to determine whether the compensation parameters always exhibit this linear trend. In addition, the structure considered here is a simple flat plate, therefore the proposed compensation approach should also be validated to more complicated structures (e.g. fuselage skin with stringers and frames) where wave reflections are also considered.

- The approach proposed here relies on one temperature measurement and has been applied only to cases where the temperature field is uniform over the structure. However, this approach is not at all limited to uniform temperature fields. The only assumption driving it is that the temperature that is being measured is a source of information that is sufficient to derive one amplitude and phase-shift linear model for each actuator-sensor path. Thus the assumption underlying the proposed method is that the temperature field (uniform or not) depends only on one variable that we are able to measure. Such temperature fields include for example the cases where the center of the plate is being heated up to a controlled and measured temperature while the border of the plate are kept to a constant temperature. Another case included within this framework is the case where one border of the plate is heated up to a controlled and measured temperature while the others are kept to a constant temperature. For future work, it could then be interesting to test the ability of the proposed method to compensate for non-uniform temperature fields described by one variable only. This also raises the question of what strategy to adopt when several temperature measurements are available. In that case, the assumption underlying the method would be that the temperature field depends only on $N$ variables that can be measured. Then, instead of building regression models that depends only on one variable, regression models that depends on $N$ variables can be built, but the core of the method is still the same.

- Although our finite element model is realistic (modeling of the structure, the glue and the piezoelectric elements), the mechanical properties of the material, the glue and the piezoelectric elements are not exactly known at elevated temperature, which make difficult the comparison between the experimental signals and the numerical ones. The numerical model allows one to perform parametric simulations (change in noise, boundary conditions, model updating for experimental baseline-free technique) that help in testing the limits of our method. The future scope of our work will include, in addition to the above-mentioned items, an updating of our finite element model using the experimental measurements. This detailed analysis will allow us to increase the knowledge about the mechanical properties of the material, the glue and the piezoelectric elements at elevated temperature which is 
important to design a robust temperature compensation model for structures under real environmental conditions.

\section{Conclusions}

This study presents a novel temperature compensation method for Lamb wave structural health monitoring. BSS is applied to i) accurately compensate changes in phase and amplitude in sensor signals due to temperature, and ii) improve the damage localization procedure at elevated temperature. The advantage of the proposed temperature compensation method is that it uses an exact analytical representation of the sensor signals through the Hilbert transform and then employs an ordinary least square (OLS) algorithm for estimating the change in amplitude and phase-shift due to temperature. The regression model used for signal reconstruction is found using the least squares method. The robustness of the proposed method is verified by the damage localization results after applying the temperature compensation model. Moreover, the result shows that it is not necessary to collect a large set of baseline sensor signals from the structure to achieve acceptable post-subtraction noise levels after compensation. The validation of the proposed temperature compensation strategy is performed on a composite plate under temperature range $\left\{16^{\circ} \mathrm{C}, 38^{\circ} \mathrm{C}, 53^{\circ} \mathrm{C}, 69^{\circ} \mathrm{C}, 85^{\circ} \mathrm{C}\right.$. Damage detection results after temperature compensation show the applicability and the effectiveness of the proposed method.

\section{Acknowledgments}

This work was partially supported by the CORALIE project.

\section{References}

Andrews J. P, Palazotto A. N, DeSimio M. P, et al. (2008) Lamb wave propagation in varying isothermal environments. Structural Health Monitoring 7(3), 265-270.

Balmes E and Deraemaeker A (2013) Modeling structures with piezoelectric materials. Theory and SDT tutorial. SDTools, Paris, France.

URL: http://www.sdtools.com/help/piezo.pdf

Chatterjee S. N (2001) Damage, stiffness loss, and failure in composite structures In Composite Structures: Theory and Practice ASTM International.

Clarke T, Simonetti F and Cawley P (2010) Guided wave health monitoring of complex structures by sparse array systems: Influence of temperature changes on performance . Journal of Sound and Vibration 329(12), $2306-2322$.

Farrar C, Duffey T, Deobling S, et al. (1999) A statistical pattern recognition paradigm for vibration-based structural health monitoring. In :2nd International Workshop on Structural Health Monitoring Stanford, CA, 8-10 September 2000 pp 76473.

Fendzi C, Mechbal N, Rébillat M, et al. (2015) A general bayesian framework for ellipse-based and hyperbola-based damage localization in anisotropic composite plates Journal of Intelligent Material Systems and Structures p. 1045389X15571383.

Garnich M. R and Akula V. M (2009) Review of degradation models for progressive failure analysis of fiber reinforced polymer composites Applied Mechanics Reviews 62(1), 010801. 
Harley J and Moura J (2012) Scale transform signal processing for optimal ultrasonic temperature compensation. Ultrasonics, Ferroelectrics and Frequency Control, IEEE Transactions on 59(10).

Ihn J and Chang F (2008) Pitch-catch active sensing methods in structural health monitoring for aircraft structures. Structural Health Monitoring 7(1), 5-19.

Kijanka P, Radecki R, Packo P, et al. (2013) GPU-based local interaction simulation approach for simplified temperature effect modelling in lamb wave propagation used for damage detection. Smart Materials and Structures 22(3), 035014.

Konstantinidis G, Drinkwater B and Wilcox P (2006) The temperature stability of guided wave structural health monitoring systems. Smart Materials and Structures 15(4), 967.

Lee S, Gandhi N, Michaels J, et al. (2011) Comparison of the effects of applied loads and temperature variations on guided wave propagation. In American Institute of Physics Conference Series Vol. 1335 pp. 175-182.

Lourakis M. I (2005) A brief description of the levenberg-marquardt algorithm implemented by levmar.

Lu Y and Michaels J. E (2005) A methodology for structural health monitoring with diffuse ultrasonic waves in the presence of temperature variations . Ultrasonics 43(9), 717 - 731.

Lu Y, Ye L and Su Z (2006) Crack identification in aluminium plates using lamb wave signals of a PZT sensor network. Smart Materials and Structures 15(3), 839.

Michaels J (2008) Detection, localization and characterization of damage in plates with an in situ array of spatially distributed ultrasonic sensors. Smart Materials and Structures 17(3), 035035.

Michaels J and Michaels T (2005) Detection of structural damage from the local temporal coherence of diffuse ultrasonic signals. Ultrasonics, Ferroelectrics and Frequency Control, IEEE Transactions on 52(10), 1769-1782.

Moll J, Schulte R, Hartmann B, et al. (2010) Multi-site damage localization in anisotropic platelike structures using an active guided wave structural health monitoring system. Smart Materials and Structures 19(4), 045022.

Montgomery D. C, Peck E. A and Vining G. G (2012) Introduction to linear regression analysis Vol. 821 John Wiley \& Sons.

Raghavan A and Cesnik C. E (2008) Effects of elevated temperature on guided-wave structural health monitoring. Journal of Intelligent Material Systems and Structures 19(12), 1383-1398.

Roweis S (1996) Levenberg-marquardt optimization.

Roy S, Lonkar K, Janapati V, et al. (2014) A novel physics-based temperature compensation model for structural health monitoring using ultrasonic guided waves. Structural Health Monitoring $\mathbf{1 3}(3), 321-342$.

Sohn H, Farrar C, Hemez F, et al. (2003) A Review of Structural Health Monitoring Literature:19962001. Los Alamos National Laboratory Report. 
Su Z and Ye L (2009) Identification of Damage Using Lamb Waves. Springer.

Wang Y, Gao L, Yuan S, et al. (2014) An adaptive filterbased temperature compensation technique for structural health monitoring. Journal of Intelligent Material Systems and Structures p. 1045389X13519001. 